ARTICLE

Received 12 Jan 2014 | Accepted 17 Mar 2014 | Published 22 Apr $2014 \quad$ DOl: 10.1038/ncomms4670

\title{
Enhancing adult nerve regeneration through the knockdown of retinoblastoma protein
}

Kimberly J. Christie ${ }^{1, \star}$, Anand Krishnan ${ }^{1, \star}$, Jose A. Martinez ${ }^{1}$, Kaylynn Purdy ${ }^{1}$, Bhagat Singh ${ }^{1}$, Shane Eaton ${ }^{1} \&$ Douglas Zochodne ${ }^{1}$

Tumour suppressor pathways may offer novel targets capable of altering the plasticity of post-mitotic adult neurons. Here we describe a role for the retinoblastoma (Rb) protein, widely expressed in adult sensory neurons and their axons, during regeneration. In adult sensory neurons, Rb short interfering RNA (siRNA) knockdown or Rb1 deletion in vitro enhances neurite outgrowth and branching. Plasticity is achieved in part through upregulation of neuronal PPARr; its antagonism inhibits Rb siRNA plasticity, whereas a PPARY agonist increases growth. In an in vivo regenerative paradigm following complete peripheral nerve trunk transection, direct delivery of Rb siRNA prompts increased outgrowth of axons from proximal stumps and entrains Schwann cells to accompany them for greater distances. Similarly, Rb siRNA delivery following a nerve crush improves behavioural indices of motor and sensory recovery in mice. The overall findings indicate that inhibition of tumour suppressor molecules has a role to play in promoting adult neuron regeneration.

\footnotetext{
${ }^{1}$ Department of Clinical Neurosciences and the Hotchkiss Brain Institute, University of Calgary, 3330 Hospital Drive NW, Calgary, Alberta, Canada T2N 4N1.

* These authors contributed equally to this work. Correspondence and requests for materials should be addressed to D.Z. (email: dzochodn@ucalgary.ca).
} 
$\mathrm{R}$ ecovery from neurological injury or disease frequently requires axon regrowth, a process that can be remarkably restrained and delayed in both central and peripheral neurons ${ }^{1}$. Less often appreciated are the considerable and persistent deficits that follow lesions of major peripheral nerve trunks, especially transection. Despite ideas otherwise, large proximal nerve lesions are associated with very poor functional recovery, rendering severe and permanent disability. Peripheral nerve injuries exceed those of the spinal cord by at least one magnitude; a complete axonal lesion at the level of the proximal sciatic nerve or brachial plexus is unlikely to be associated with the recovery of foot or hand function, respectively ${ }^{2-4}$.

As might be expected from such poor outcomes, regrowth of axons across transection gaps, common forms of injury, is highly constrained and limited. Indeed, only $10 \%$ of axons from the proximal stump may eventually successfully navigate to their targets ${ }^{5}$. Reluctant regrowth of axons may be partially responsive to neuronal and pleiotropic growth factors, depending on their receptor expression. Some of these factors are already present at injury sites. Alternatively, intrinsic mechanisms that brake more robust outgrowth paradoxically exist within neurons, a role that likely ensures stability of complex connections in adults. After injury, the ongoing expression of these molecular 'brakes' attenuates regrowth. Examples include RhoA GTPase and $\mathrm{PTEN}^{6,7}$. A related issue is that signalling pathways influencing overall axon outgrowth and neuron plasticity overlap with those associated with protection and survival. Two examples include PI3K-Akt signalling downstream of many growth factors that supports both neuronal survival and axon outgrowth and HSP27, a molecular chaperone that similarly protects neurons and enhances regeneration ${ }^{8,9}$. 'Brake' or plasticity molecules in neurons also overlap with those that influence tumour growth; especially recognized are PTEN and PI3K. It is thus plausible that other molecules and pathways capable of altering tumour growth and that are normally expressed in stable adult neurons might offer new avenues for manipulating neurological regenerative growth.

In this work we identify a classical tumour suppressor pathway involving the protein retinoblastoma $(\mathrm{Rb})$ as a regulator of peripheral nerve regeneration. $\mathrm{Rb}$ offers opportunities to promote growth through promiscuous interactions involving a number of signalling networks that are involved in growth stimulation and have an impact on survival and apoptosis ${ }^{10}$. Importantly, these networks suggest that $\mathrm{Rb}$ might operate downstream of the canonical survival pathways of Raf-MEK and PI3K-Akt. Not previously explored in the adult nervous system, we show that $\mathrm{Rb}$ is widely expressed and operates to suppress neuronal plasticity. Its knockdown is associated with enhanced plasticity that translates into a robust action on local axons and their accompanying Schwann cells (SCs) during regrowth from complete nerve trunk transection. The overall findings highlight the possibilities that manipulation of tumour suppressor pathways, not routinely considered in post-mitotic neurons, might be used to enhance adult nervous system regeneration.

\section{Results}

Adult sensory neurons express the $\mathbf{R b}$ protein. A role for the $\mathrm{Rb}$ protein, a key inhibitor of cell cycle progression, commonly deleted in human tumours, has not been described in the adult nervous system. During development, $\mathrm{Rb}$ may have critical roles in neuronal precursor cell proliferation and neuron migration; however, it has been uncertain whether $\mathrm{Rb}$ expression persists within or has an impact on adult neurons ${ }^{11,12}$. Given these uncertainties, we observed its expression in a large proportion of dorsal root ganglion (DRG) sensory neurons, colabelling with neurofilament expressing large calibre neurons but also present in neurofilament-poor smaller calibre neurons (Fig. 1). These patterns of expression suggested the possibility of a widespread functional role. Expression was largely neuronal within the cytoplasm and prominently displayed in nuclei. In peripheral nerve trunks, $\mathrm{Rb}$ was expressed almost exclusively in axons, colabelling with a neurofilament label but with relatively minor colabelling of SCs (Fig. 2). Following injury, Rb was robustly expressed in axons at the injury site. SCs at the regenerative nerve front were not prominently labelled (Fig. 2). S100 $\beta$ SC profiles surrounded the axonal $\mathrm{Rb}$ profiles. Distal to the site of injury, $\mathrm{Rb}$ was identified in profiles undergoing axonal degeneration, indicating its presence in phagocytic SCs and macrophages. After injury, Rb expression in DRG sensory neurons and in axons persisted, although there was a relative decline in its mRNA and protein levels (Fig. 1; Supplementary Figs 1 and 2). Overall, the findings supported the possibility that $\mathrm{Rb}$ was poised to influence neuronal behaviour after injury.

Rb protein knockdown enhances neuron plasticity. To address the potential impact of $\mathrm{Rb}$ expression on adult sensory neurons, we analysed their outgrowth patterns in response to Rb knockdown. This approach has predicted in vivo behaviour in response to several plasticity signals studied in previous work including RHOA and PTEN inhibition ${ }^{6,7}$. Since $\mathrm{Rb}-/-$ mice are embryonically lethal, suitable adult populations of their neurons are not available. Similarly, the phenotype of $\mathrm{Rb}+/-$ mice is highly impaired and abnormal, limiting the health and viability of their neurons ${ }^{13}$. In view of these concerns, as well as our intent to temporarily and locally knockdown an oncogenic molecule, we examined the impact of transient knockdown on Rb using siRNA directed specifically to neurons.

First, we examined adult sensory neurons in dissociated cell culture using a lipid transfection protocol that achieves robust and predictable mRNA knockdown. We confirmed that two specific but differing Rb siRNA constructs were extensively taken up by neurons and suppressed their $\mathrm{Rb}$ mRNA and protein (Fig. 3). No impact of scrambled sequence control siRNA was identified. Pre-injured adult sensory neurons, with an already enhanced baseline growth rate, and designed to mimic responses to in vivo injury, were exposed to $\mathrm{Rb}$ or scrambled siRNA for $18-20 \mathrm{~h}$. Their pattern of neurite outgrowth was analysed. Rb-suppressed neurons with siRNA uptake showed an overall increase of over $50 \%$ in neurite outgrowth and maximum process length. In addition, there was a $60 \%$ rise in numbers of neurite branches (Fig. 3e-h). Neurons that exhibited increased plasticity were specifically those that acquired the labelled $\mathrm{Rb}$ siRNA construct (Fig. 3i,j). The findings identified an added impact of $\mathrm{Rb}$ suppression that was superimposed on 'preconditioned' accelerated outgrowth of pre-injured adult neurons. To identify whether the Rb1 siRNA knockdown strategy had an impact on growth in a separate rodent species of adult sensory neuron, we repeated the analysis in uninjured DRG neurons of adult mice exposed to Rb1 siRNA or scrambled controls. The approach similarly identified a robust impact on neurite outgrowth and branching (Supplementary Fig. 3). The addition of siRNA to E2F1, a critical target of Rb, eliminated the impact of Rb1 siRNA on outgrowth and branching in rat DRG neurons, indicating a role for E2F1 in neuronal plasticity (Supplementary Fig. 4). Taken together, these findings indicated a robust role for $\mathrm{Rb}$ in suppressing neurite outgrowth, reversed by local knockdown.

As a further validation of the impact of $\mathrm{Rb}$ knockdown on the plasticity of sensory neurons, we harvested lumbar and thoracic DRG neurons from adult Rb1-floxed mice (FVB; $129-R b 1^{t m 2 B r n}$ ) and transfected them with control adenovirus (Ad-GFP (greenfluorescent protein)) or Cre recombinase expressing adenovirus 

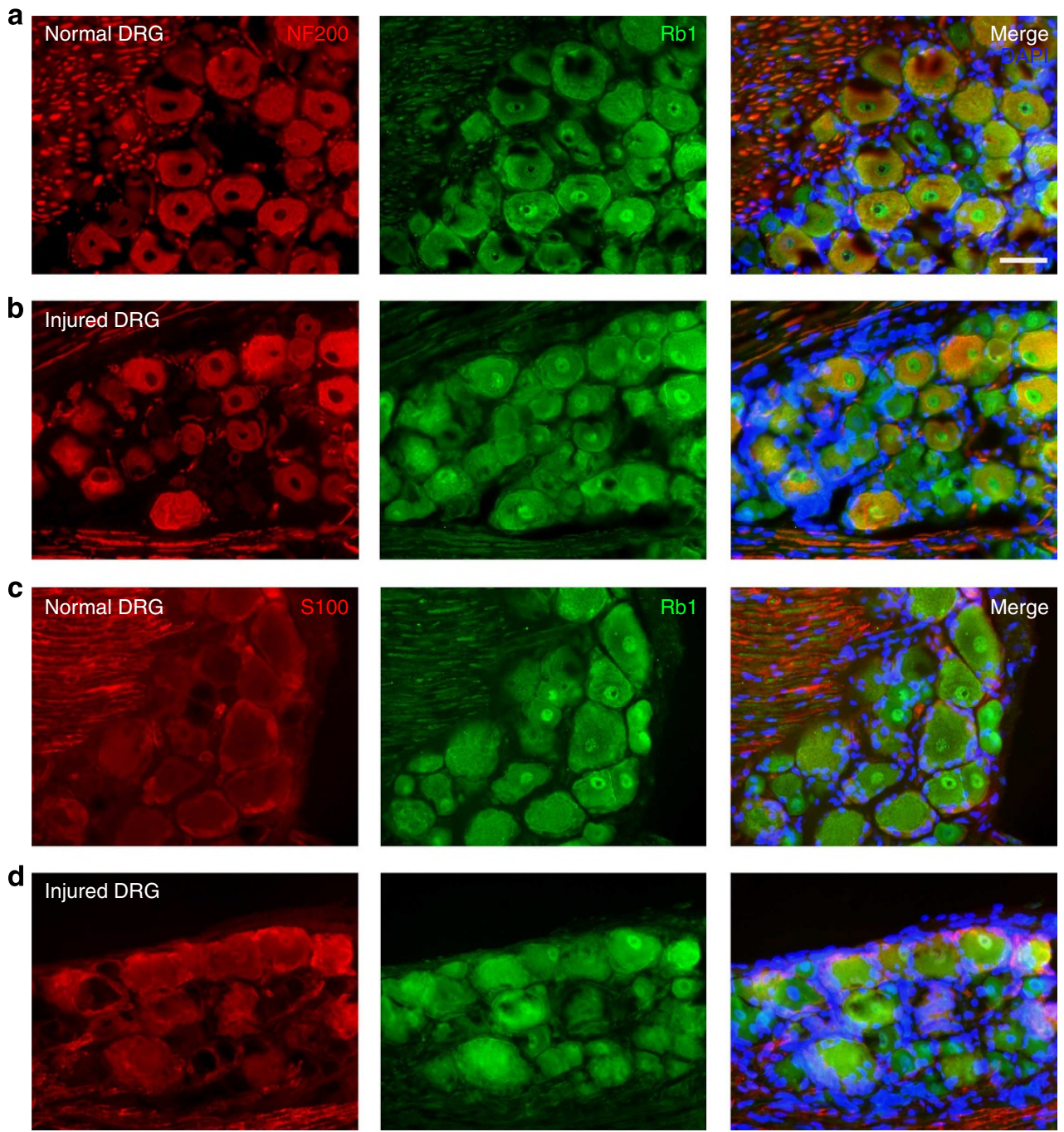

Figure 1 | Rb1 is expressed in sensory DRG. Transverse sections of normal adult DRG with co-expression in neurons of NF200 and Rb1, the latter in the cytoplasm and nuclei (a). Transverse section of 3d injured DRG with co-expression of NF200 and Rb1 (b). There is relatively little co-expression of Rb1 and S100 $\beta$, a marker of Schwann cells, in DRG (c); As in c, following 3d axotomy injury (d). Bars $=50 \mu \mathrm{m}$.

(Ad-CMV (cytomegalovirus)-Cre) particles. In separate experiments the cells were infected with Ad-GFP-2A-iCre particles, which express both Cre recombinase and GFP. Neurons transfected with Ad-GFP-2A-iCre are illustrated in Fig. 4. Rb knockdown was confirmed using qRT-PCR. As above, the patterns of neurite outgrowth were compared between neurons with Rb1 knockdown and those exposed to virus without Cre recombinase. Neurons with $\mathrm{Rb}$ knockdown had robust rises in neurite outgrowth, maximum process length, numbers of processes and numbers of branches (Fig. 4; Supplementary Fig. 4).

Since $\mathrm{Rb}$ knockdown has been linked to evidence of apoptosis in some published models ${ }^{11}$, we analysed the levels, in neurons exposed to Rb knockdown or deletion, of phosphohistone H2A.X and activated caspase 3, putative markers of DNA doublestranded break and apoptosis, respectively (Fig. 5a-c; Supplementary Fig. 4). We found no difference in the levels of phosphohistone H2A.X in the control and Rb siRNA-treated cells. Whereas baseline levels of phosphohistone H2A.X and activated caspase 3 were slightly higher, qualitatively, in adenovirus-exposed neurons, levels were similar in neurons treated with Cre recombinase compared with controls. Moreover, the presence of these molecular markers did not correlate with any morphological evidence of impaired viability; processes were intact and had adequate branching capability. Expression of activated caspase 3 has been previously described in intact and viable DRG neurons ${ }^{14}$. As a quantitative measure of cell death, we used an lactate dehyrogenase $(\mathrm{LDH})$ release toxicity assay and compared $\mathrm{Rb}$-floxed neurons from mice transfected with Cre to control transfections and also compared rat neurons exposed with Rb1 siRNA to those exposed to scrambled sequence control siRNA. Neither Rbl knockdown strategy was associated with $\mathrm{LDH}$ release that exceeded controls, indicating an absence of significant cell death (Fig. 5d). Taken together, these results did not identify evidence of cell death associated with $\mathrm{Rb}$ knockdown in the time frames and conditions examined here. Selective loss of neurons did not account for the readouts identifying substantial rises in neurite outgrowth identified in our system.

Rb knockdown-related targets. We next examined several candidate plasticity proteins that are known targets of Rb. Neurons undergoing $\mathrm{Rb}$ knockdown were harvested and protein lysates were examined. Pak1, CDK5 and PPAR $\Upsilon$ have previously been reported to be downstream effectors of $\mathrm{Rb}$ in multiple systems and, interestingly, these molecules were shown to have possible 

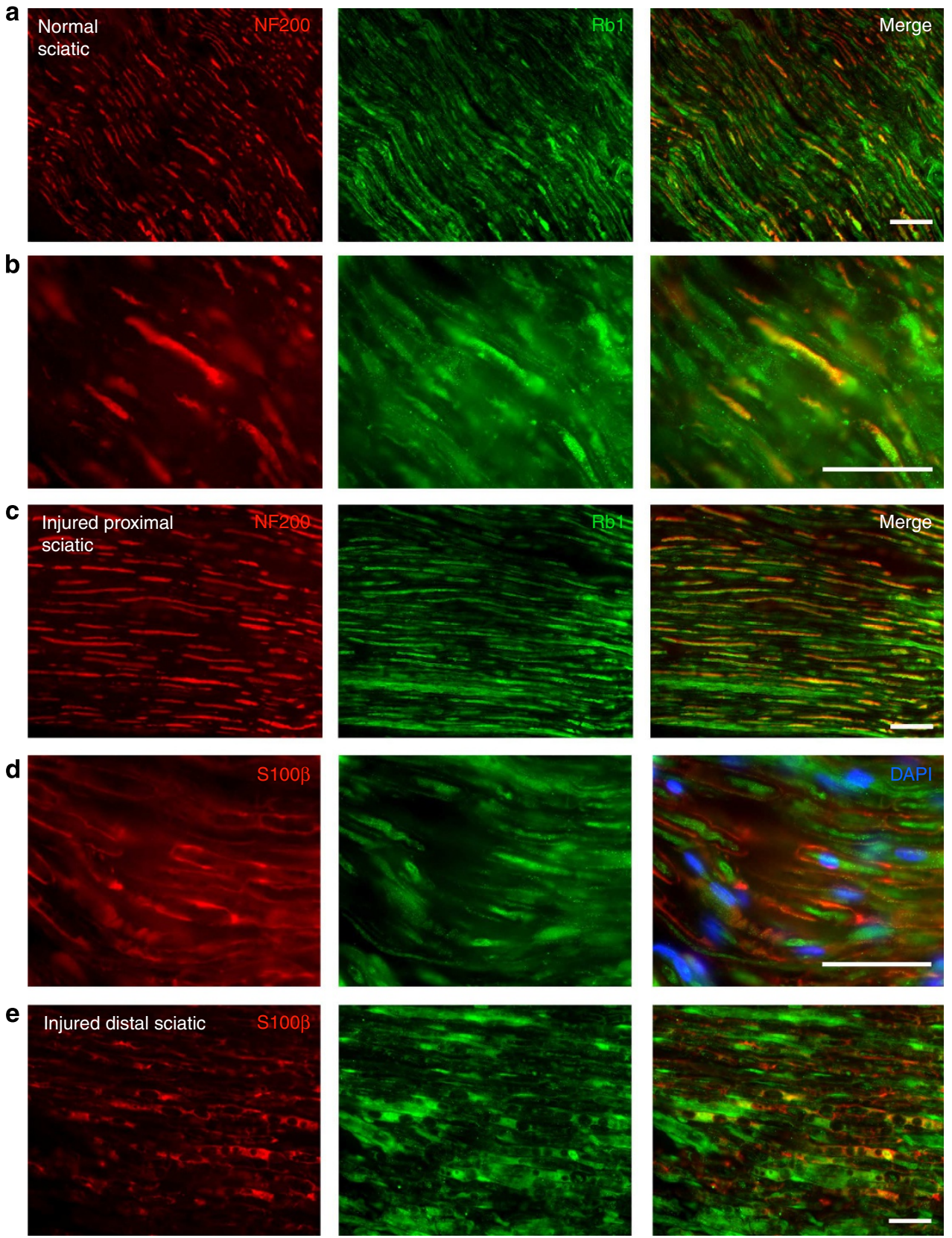

Figure $\mathbf{2}$ | Rb1 is preferentially expressed in neurons and axons. Expression of Rb1 in normal sciatic nerve (a). Note the colabelling with a neurofilament marker indicating expression in axons. Expression at higher power (b). Longitudinal section of $3 d$ injured sciatic nerve proximal to the injury zone, with intense co-expression of Rb1 in NF200 labelled axons (c). Lack of co-expression of S100 $\beta$ and Rb1 in the proximal tip of injured sciatic nerve (d). Note that the SC S100 $\beta$ surrounds the Rb1 axon profiles. Limited co-expression of S100 $\beta$ and Rb1 in the distal segment of injured sciatic nerve, largely involving axons undergoing axonal degeneration (e). Bars $=50 \mu \mathrm{m}$.

roles on nerve regeneration ${ }^{15-20}$. Particular consideration was given to the PI3K-Akt pathway, well known to enhance outgrowth in response to insulin, other growth factors and PTEN inhibition ${ }^{9,21}$ (Fig. 6; Supplementary Fig. 5a,b). Changes in the levels of pAkt however, the key effector of this pathway, were not identified. Pak1 is a second messenger for Rac1, a GTPase associated with increased growth cone extension. Its levels were not altered by $\mathrm{Rb}$ knockdown. Finally, we did not detect a significant change, beyond a mild trend towards downregulation, in CDK5, a neuronal specific cyclin-dependent kinase. CDK5/p35 may operate upstream of $\mathrm{Rb}^{22}$. In contrast to these results, we identified an unexpected but consistent upregulation of PPAR $Y$ protein and mRNA in sensory neurons following knockdown of
$\mathrm{Rb}$ (Fig. 6b-i). This change was verified through two differing siRNA constructs. We next confirmed using immunocytochemistry that PPAR $\Upsilon$ was expressed in adult sensory neurons, located in their cytoplasm, nucleus and to a lesser extent in their neurite processes (Fig. 6i). After axotomy injury, there was a nonsignificant trend towards its upregulation (Supplementary Figs 1 and 2). Overall, the findings identified a new neuronal target, PPAR $\Upsilon$, in adult neurons having undergone $\mathrm{Rb}$ knockdown.

PPARY contributes to Rb knockdown plasticity. To test the potential significance of PPAR $\Upsilon$ upregulation, we examined 

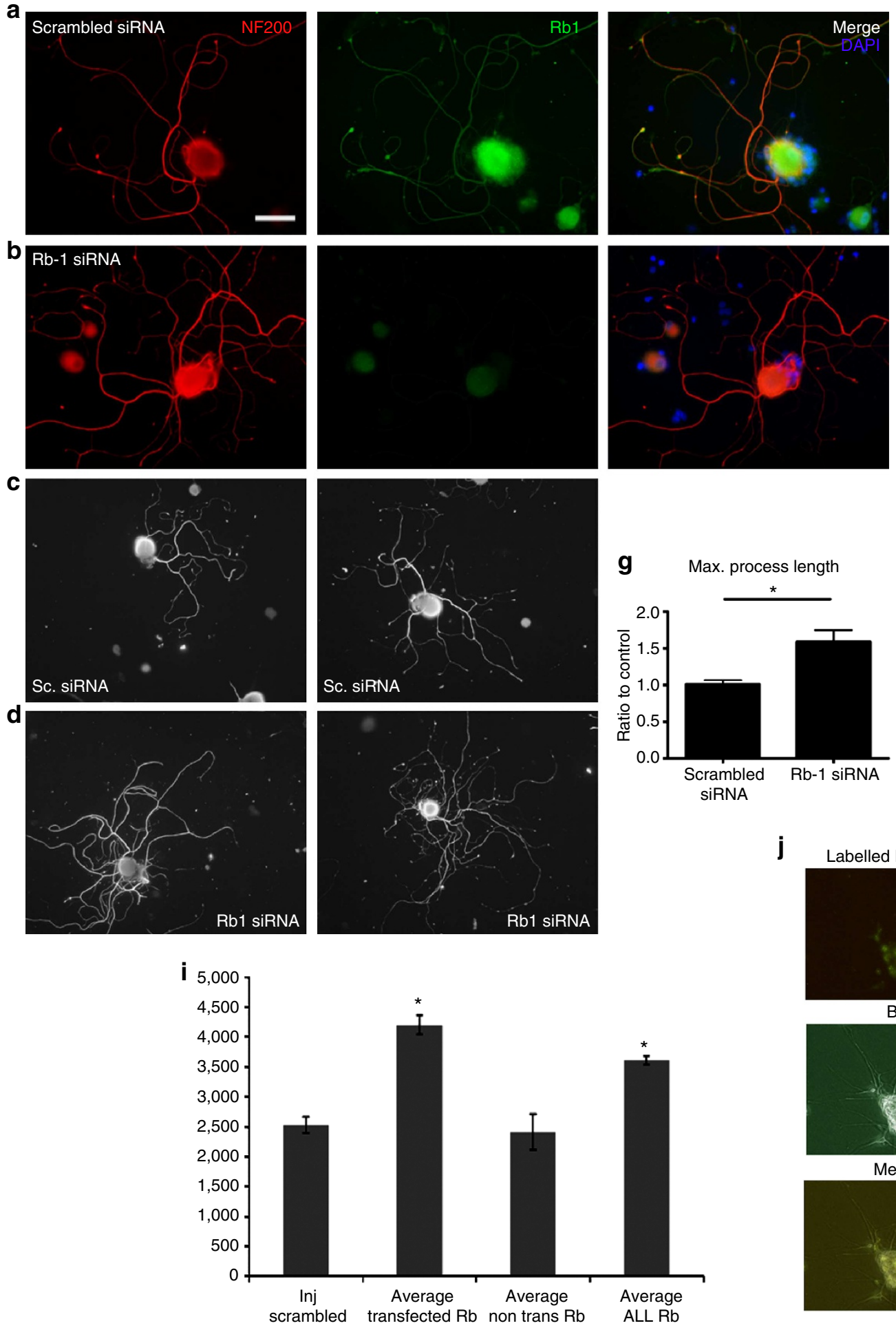

Figure 3 | siRNA knockdown of Rb1 increases DRG neurite outgrowth. Rb1 is co-expressed with NF200 in injured and dissociated adult DRG neurons (a). Knockdown of Rb1 with an siRNA decreases Rb1 expression (b). Images of DRG neurons with scrambled siRNA (c). Images of DRG neurons with Rb1 siRNA-note the increase in neurite outgrowth $(\mathbf{d})$. (Bar $=50 \mu \mathrm{m})$. Rb1 siRNA significantly decreases Rb1 mRNA $\left({ }^{\star} P<0.001, n=4 ;\right.$ unpaired two-tailed Student's t-test) (e). Neurite outgrowth quantified from MetaXpress, represented as a ratio in transfected neurons to control (Scrambled: $2,486 \pm 80 \mu \mathrm{m}$, Rb1: $4,310 \pm 114 \mu \mathrm{m},{ }^{\star \star} P<0.0001, n=5$; unpaired two-tailed Student's $t$-test) (f). Maximum process length (Scrambled: 1,258 $\pm 71 \mu \mathrm{m}, \mathrm{Rb} 1$ : $1,970 \pm 140 \mu \mathrm{m},{ }^{\star} P<0.01, n=5$; unpaired two-tailed Student's $t$-test) (g). Number of branches (Scrambled: $100 \pm 6, R b 1: 160 \pm 12,{ }^{\star} P<0.05, n=5$; unpaired two-tailed Student's t-test) (h). Values are means \pm s.e.m. Quantitation of total neurite outgrowth analysed in sensory neurons in which labelled Rb1 siRNA was specifically identified within the neuron or in all sensory neurons exposed in the culture dish to Rb1 siRNA (i). Note that enhanced neurite outgrowth is specifically associated with neurons in which visualization of labelled siRNA uptake was confirmed (scrambled versus average transfected, or average ALL Rb ${ }^{\star} P<0.0001$; unpaired two-tailed Student's $t$-test). Labelled siRNA within harvested dissociated adult sensory neurons identified by brightfield (BF) and fluorescence (j). 

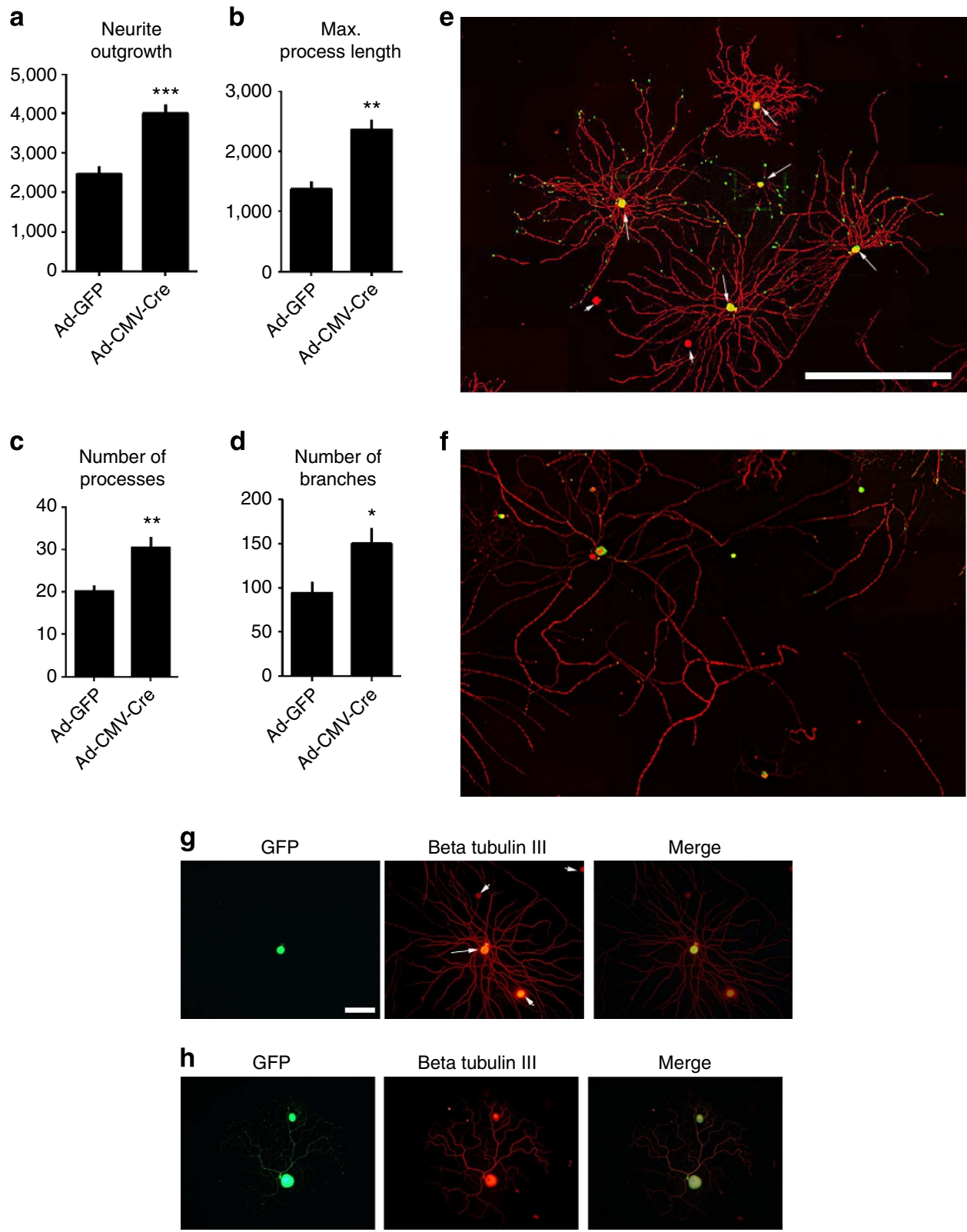

Figure 4 | Conditional knockdown of Rb1 in adult sensory neurons from Rb1-floxed mice is associated with enhanced neurite outgrowth. Conditional knockdown of Rb1 is associated with rises in neurite outgrowth, maximum process length, numbers of processes (neurites) and numbers of branches (a-d) $\left({ }^{\star \star \star} P<0.001\right.$ for $\mathbf{a},{ }^{\star \star} P=0.001$ for $\mathbf{b}$, ${ }^{\star \star} P<0.01$ for $\mathbf{c},{ }^{\star} P<0.05$ for $\mathbf{d}$; $n=6 /$ group; unpaired two-tailed Student's $t$-tests). Values are means \pm s.e.m. Low-power image of neurite outgrowth in Ad-GFP-2A-iCre-infected cells. Neurons are labelled with beta III tubulin and viral transfection indicated by a GFP-Cre-expressing viral construct. Long arrows indicate Cre recombinase-expressing cells and short arrows indicate Cre recombinase non-expressing cells. Note the lesser neurite outgrowth in cells without GFP-Cre (e). Low-power image of neurite outgrowth in Ad-GFP (control)-infected cells. Outgrowth was less extensive in this neuronal population (f) (Bar $=500 \mu \mathrm{m}$ for $\mathbf{e}, \mathbf{f})$. High-power image of neurite outgrowth in Ad-GFP-2A-iCre-infected cells. Long arrows indicate Cre recombinase expressed cells and short arrows indicate Cre recombinase non-expressed cells $(\mathbf{g})($ Bar $=100 \mu \mathrm{m}$ for $\mathbf{g}, \mathbf{h})$.

Higher-power image of neurite outgrowth in Ad-(control)-infected cells (h).

neurite outgrowth and branching in neurons with combined $\mathrm{Rb}$ knockdown and PPAR $\Upsilon$ antagonism. GW9662, a selective competitive pharmacological antagonist of PPAR $\Upsilon$ dosedependently inhibited the enhancement of neurite outgrowth associated with Rb siRNA (Fig. 7a-e; Supplementary Fig. 6). Outgrowth inhibition included total neurite outgrowth, numbers of primary neurites, the longest neurite measured and the number of neurite branches. At its higher dose, GW9662 suppressed outgrowth values below those of controls, suggesting that its constitutive actions might be important for baseline neuron growth. In contrast to the suppression of growth by the PPARY antagonist, a PPAR $\Upsilon$ agonist, 15PGJ2, at $100 \mathrm{nM}$, increased overall outgrowth and the length of the longest neurites (Fig. 7f). The impact of the agonist, however, was less robust than that of $\mathrm{Rb}$ knockdown and did not include an impact on neurite branching. Only nonsignificant trends towards increased branch numbers were observed (Fig. 7f). Taken together, the findings provided evidence that PPARY acts downstream of $\mathrm{Rb}$ and may contribute towards plasticity when its repression by $\mathrm{Rb}$ is relieved. 

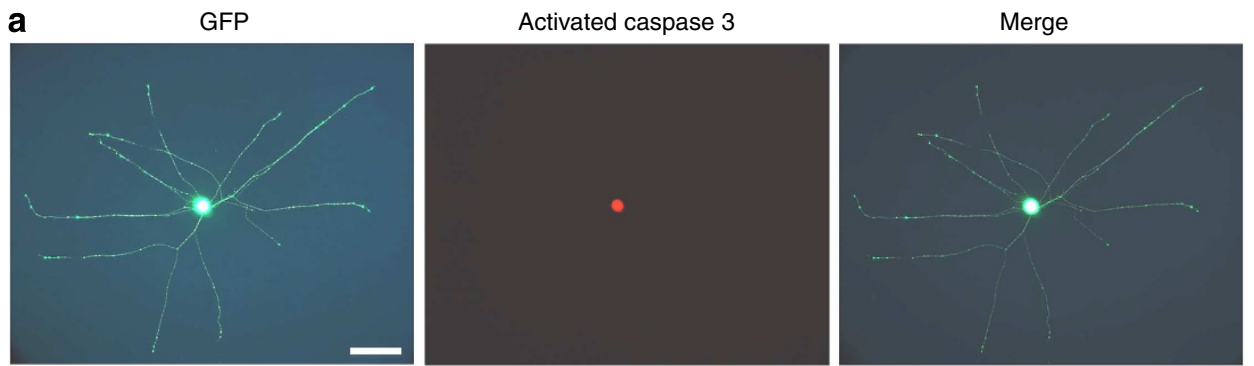

b

GFP
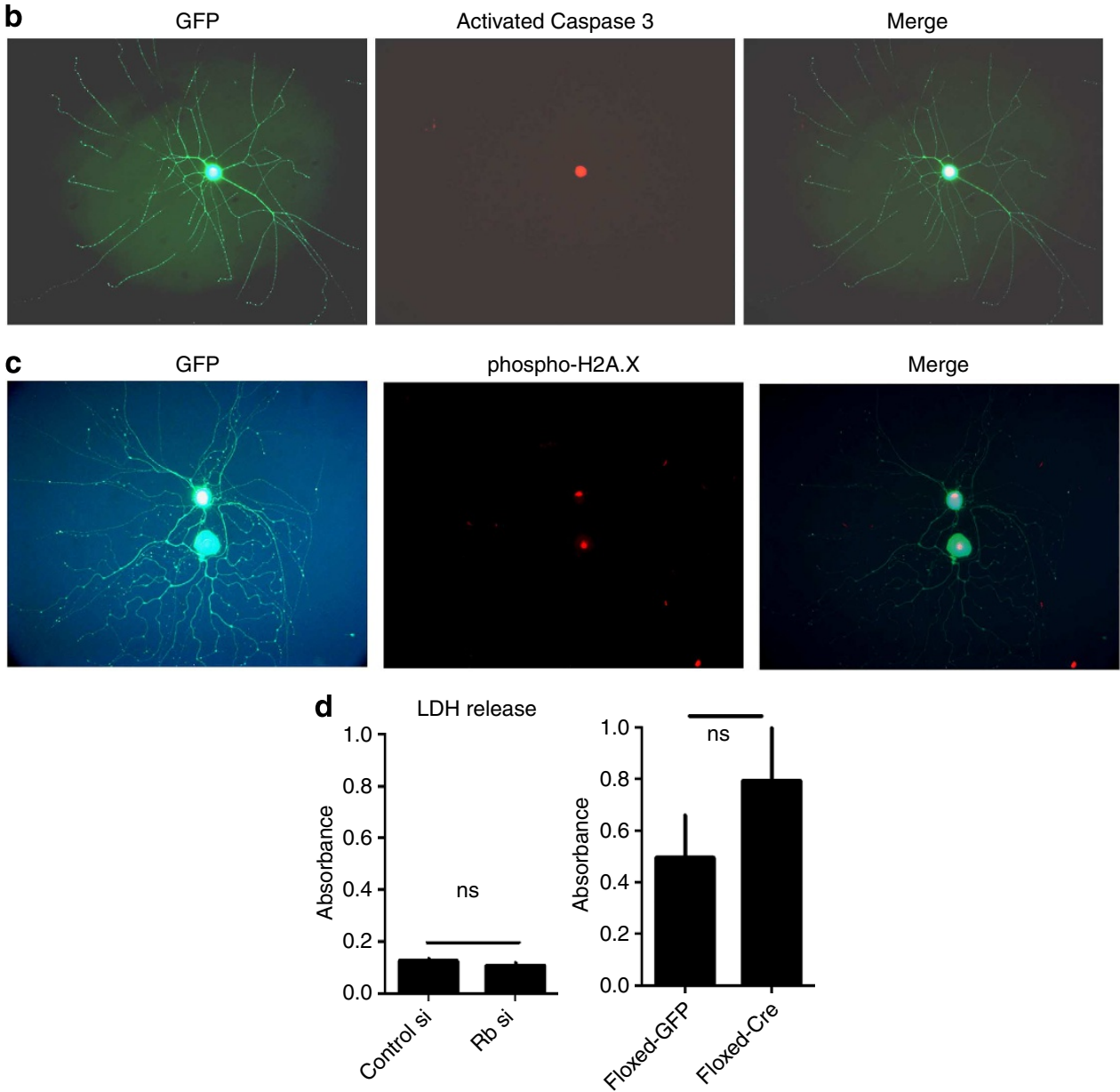

Figure $\mathbf{5}$ | Transient knockdown of Rb in sensory neurons is not associated with increased cell death. While activated caspase 3 expression was observed in neurons in both control (Ad-GFP) (a) and Cre-expressing (Ad-GFP-2A-iCre) (b) treated cells, neurons had normal morphology and intact neurite outgrowth. Expression of activated caspase 3 has been previously reported in significant numbers of intact and viable neurons ${ }^{14}$. Expression of phospho-H2A.X in Ad-GFP-2A-iCre-treated cells (c) was also associated with intact and viable neuron morphology (and see Supplementary Fig. 4)

(Bar $=100 \mu \mathrm{m}$ a-c). LDH release (d) was similar (ns; $n=4$, rats; unpaired two-tailed Student's $t$-test) in adult rat DRG neurons exposed to scrambled siRNA compared with Rb siRNA (as in Fig. 3 ) and in adult mouse Rb-floxed neurons ( $n s ; n=3$; unpaired two-tailed Student's $t$-test) exposed to control adenovirus compared to adenovirus expressing Cre recombinase. Values are means \pm s.e.m.

Rb knockdown improves outgrowth after axotomy. To test whether $\mathrm{Rb}$ knockdown might be associated with altered behaviour of regenerating axons in vivo, we examined their outgrowth properties following exposure to local administration of siRNA delivered to injured nerve trunks. In previous studies we have shown that local nonviral delivery of siRNAs provides specific and potent actions both at the site of application but also through retrograde uptake by neurons, when applied to outgrowing axons and their accompanying SCs $s^{7,23,24}$. Despite assumptions otherwise, transected peripheral neurons are highly hesitant in their outgrowth properties and limited to slow and staggered advancements beyond the injured zone ${ }^{1}$. Only $10 \%$ of axons are estimated to successfully cross these types of injury zones, even with microfascicular repair ${ }^{5}$. An improvement in this success rate could translate into improved regenerative outcomes.

After transection of the sciatic nerves in adult rats, a gap of $3-5 \mathrm{~mm}$ is formed from retraction of the distal and proximal stumps, similar to clinical scenarios in human nerve injuries. In our experimental paradigm, a silicone conduit was inserted within the retraction gap and connected to the proximal and distal stumps, as used in clinical nerve repair. A side arm of the conduit was connected to a subcutaneous access port to allow repeated installation of local siRNAs into the regenerative microenvironment, and analysis was carried out of the extent of 
a

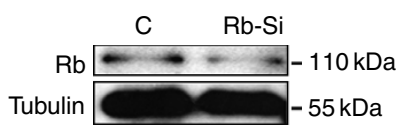

b

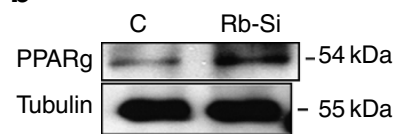

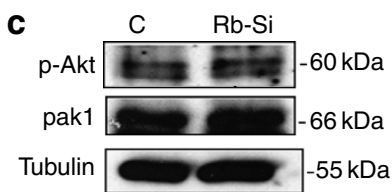

d

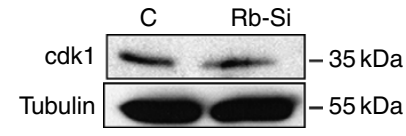

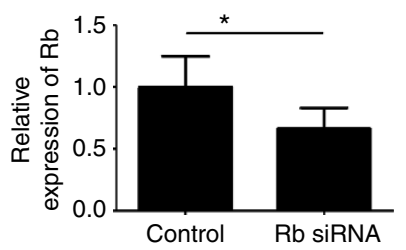

f

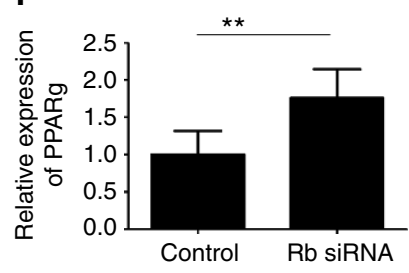

g

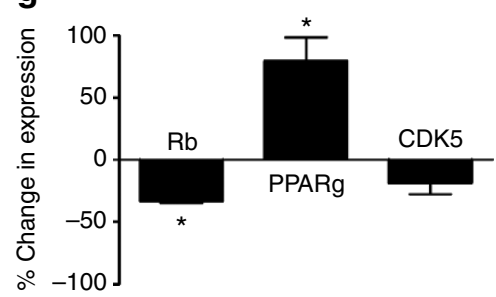

h

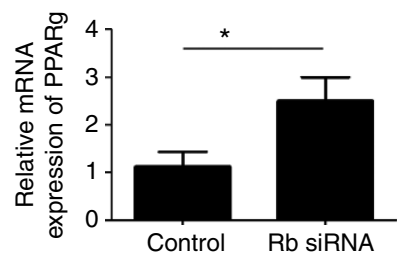

i
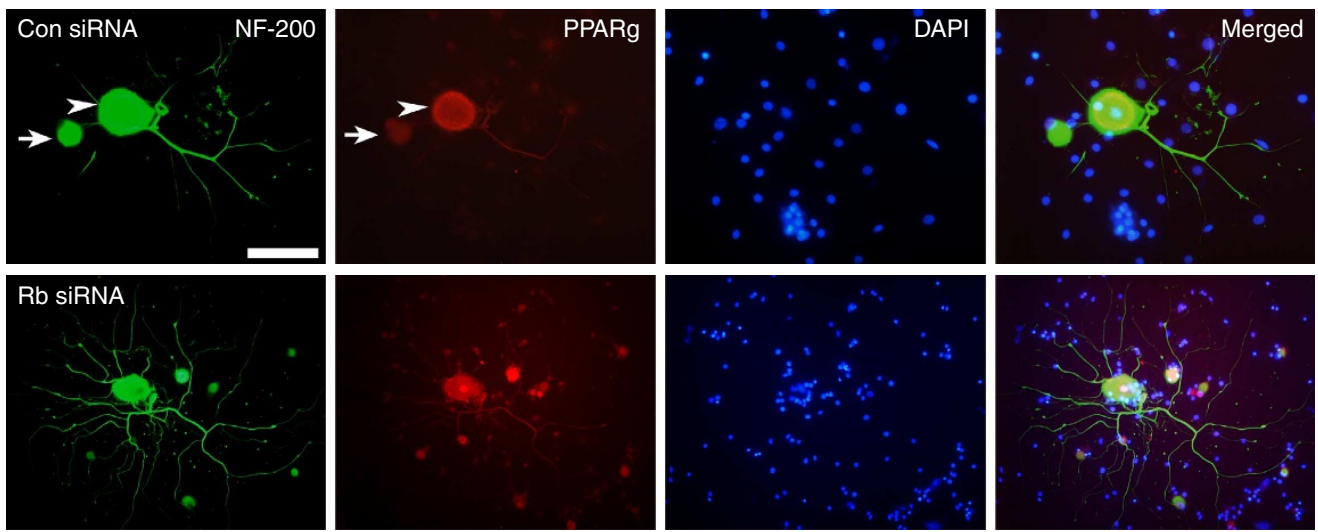

Figure 6 | Knockdown of Rb1 increases the expression of PPARY. Rb1 siRNA is associated with knockdown of the Rb1 protein in dissociated adult sensory neurons (a) and a rise in PPARY expression (b) without changes in pAkt, Pak1 or CDK5 (c,d). Quantitation of western blot data showing declines in $\mathrm{Rb}(\mathbf{e})\left({ }^{\star} P<0.05 ; n=4\right.$; paired two-tailed Student's $t$-test) and rises in PPARY(f) $\left({ }^{\star \star} P<0.01 ; n=4\right.$; paired two-tailed Student's $t$-test) and relative changes in expression ( $\mathbf{g})$. Rb siRNA was also associated with a rise in PPAR $\mathrm{mRNA}(\mathbf{h})\left({ }^{\star} P=0.048 ; n=\right.$ four control/scrambled, three Rb siRNA; unpaired Mann-Whitney two-tailed test). Values are means \pm s.e.m. Images (i) of dissociated adult sensory neurons exposed to scrambled (Con, top panels) or Rb siRNA and labelled with antibodies to neurofilament (green,NF200), PPARY (red) and colabelled with a nuclear stain (blue, DAPI). Note the greater neurite outgrowth of neurons exposed to Rb siRNA and the qualitative increase in PPARY-staining intensity. Bar $=100 \mu \mathrm{m}$.

axon outgrowth from the proximal stump (Fig. 8a,b). Both the approach and the impact of this strategy have been extensively validated in previous work $\mathrm{k}^{25,26}$. After transection and conduit placement, injections of $0.2 \mathrm{ml}$ were given on days $0,1,3$ and 5 for $\mathrm{Rb}$ or scrambled siRNA ( $2 \mu \mathrm{g}$ per injection). Bridges were processed for immunohistochemistry and probed for the axon marker NF200 (1:800) and a marker for accompanying activated SCs, glial fibrillary acidic protein (GFAP; 1:250). At the beginning of the outgrowth zone in the first separate high-power field $(400 \mu \mathrm{m})$, a clear demarcation could be recognized in the overall structure of the nerve trunk, and the onset of regenerative neurofilament containing axon profiles. Starting at the distal edge of the proximal stump, we counted profiles in a line perpendicular to the direction of the bridge in serial fields distally in high-power fields every $270 \mu \mathrm{m}$ until reaching the last field expressing the heavy-chain neurofilament protein, NF200 (neurofilament; Fig. 8b-d).

As with the in vitro experiments, local in vivo siRNA was associated with knockdown of Rb1 siRNA within the axon outgrowth injury zone (Fig. 8e). In response to knockdown we observed a substantial rise in the numbers of outgrowing axons in most fields distal to the transection zone as well as a greater outgrowth distally (Fig. 8c,d,f,h). The outgrowth in proximal fields indicated a vigorous sprouting response while individual axons also extended greater distances into the conduit. A separate analysis of SC outgrowth similarly confirmed substantially larger numbers of profiles in both proximal and distal fields beyond the transection zone (Fig. 8g,i). Overall, these findings extended the observations in vitro that $\mathrm{Rb}$ knockdown can be translated into a meaningful and robust in vivo paradigm, with a substantial impact on outgrowth. The findings also confirm our previous conclusions that closely knit axon-SC partnerships allow strategies that ostensibly only support one or other of these partners to ultimately influence growth in both of them ${ }^{24,25}$.

Rb knockdown improves recovery from nerve injury. We next asked whether in vitro axon sprouting and early in vivo outgrowth, as demonstrated above, translate into longer-term recovery from nerve injury. We examined behavioural indices of sensory and motor axon regrowth in mice following a sciatic nerve crush injury with or without $\mathrm{Rb}$ knockdown using local administration of siRNA. Mice were chosen for these experiments because their shorter nerve trajectories allow more rapid evaluation of recovery of function. We also aimed to determine whether the putative impact of $\mathrm{Rb}$ knockdown could be demonstrated in a second, separate animal model. siRNA (Rb1 or scrambled) solution was applied to the crush area immediately after crush then serially for 5 days both at the crush site and into 
a

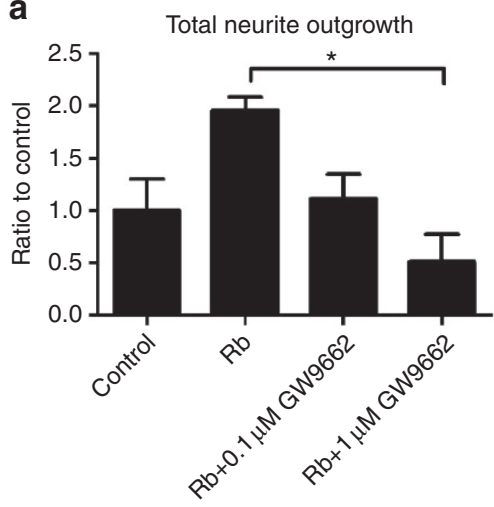

d

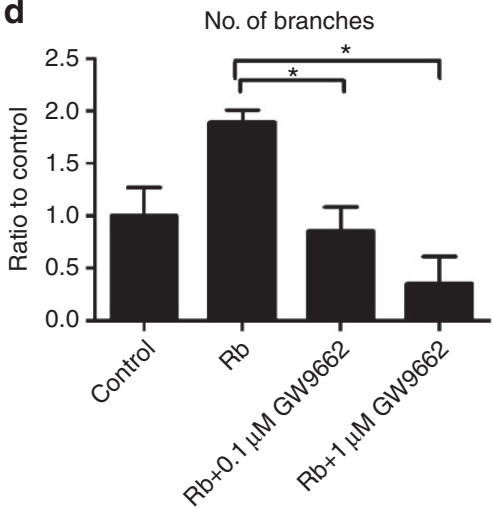

e

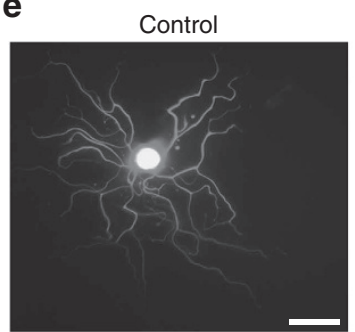

b

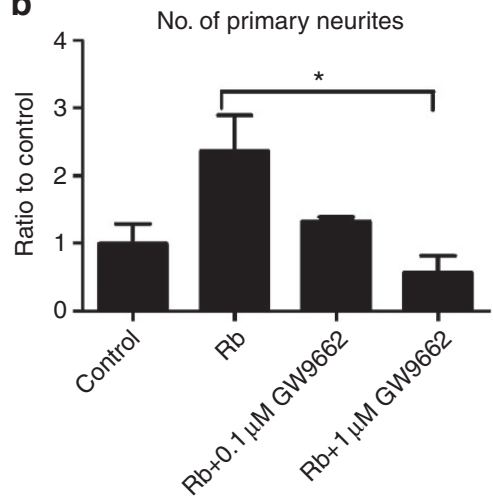

C

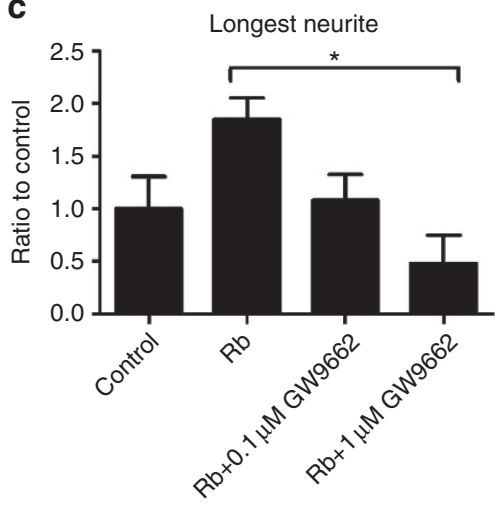

$\mathbf{f}$

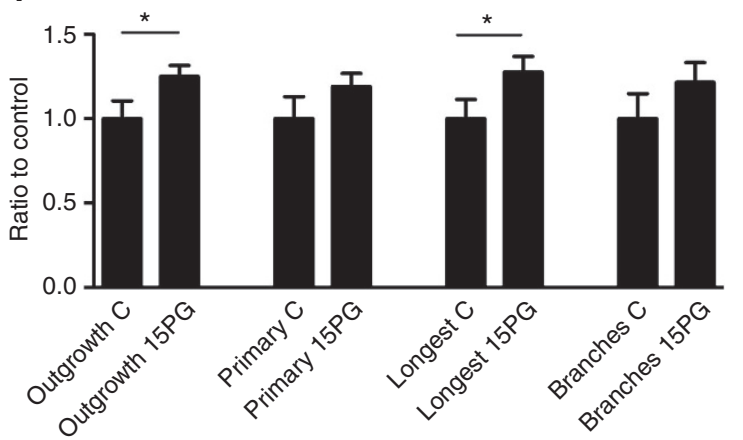

$\mathrm{Rb}$
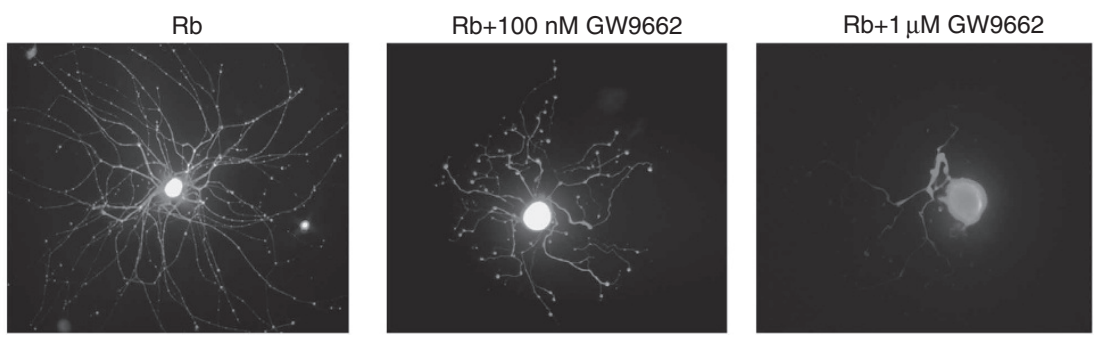

Figure 7 | Neurite plasticity following Rb1 knockdown is blocked by a PPARY antagonist. Dissociated uninjured (non-preconditioned) adult sensory neurons exposed to one of two differing Rb1 siRNA constructs showed enhanced growth. Bars indicate control neurite outgrowth, Rb1 siRNA outgrowth and Rb1 siRNA outgrowth with GW9662 at 0.1 or $1.0 \mu \mathrm{M}$. Rb1 siRNA is associated with rises in total neurite outgrowth (a) ${ }^{\star} P<0.05 ; n=3 ;$ analysis of variance $P<0.05$; post hoc Tukey), greater numbers of primary neurites (b) ( ${ }^{\star} P<0.05 ; n=3$; ANOVA $P<0.05$; post hoc Tukey), longer neurites (c) $\left({ }^{\star} P<0.05 ; n=3\right.$; ANOVA $P<0.05$; post hoc Tukey) and rises in the number of neurite branches (d) ( $P<0.05 ; n=3 ;$ ANOVA $P<0.01 ;$ post hoc Tukey). The panel below (e) illustrates examples of sensory neurons exposed to an Rb1 siRNA constructs without or with two differing doses of GW9662 $($ Bar $=10 \mu \mathrm{m}, \mathrm{Bar}=100 \mu \mathrm{m})$. In contrast, exposure of dissociated adult sensory neurons to a PPARY agonist (f), 15PGJ2 at $100 \mathrm{nM}$ was associated with rises in total neurite outgrowth $\left({ }^{\star} P<0.01 ; n=7\right.$; paired two-tailed Student's $t$-test) and in the length of the longest neurite $\left({ }^{\star} P<0.05 ; n=7 ;\right.$ paired two-tailed Student's $t$-test). Values are means \pm s.e.m.

the distal territory of the nerve in the hindpaw. We confirmed that this approach, also described in previous work, knocked down the $\mathrm{Rb}$ mRNA transcript both locally within the injured nerve and in the ipsilateral DRG (there was a nonsignificant trend towards increased PPARY mRNA in the DRG (Supplementary Fig. 4)). As demonstrated using other siRNA constructs without viral delivery, DRG knockdown occurs from retrograde transport of the siRNA ${ }^{24}$. Measures of hindpaw grip strength, mechanical sensitivity and thermal sensitivity were carried out at baseline and then repeated at 14 and 28 days. By 14 days mice exposed to scrambled siRNA had lower hindpaw grip strength than preinjury and also had mechanical hypoalgesia and thermal hyperalgesia, all expected features of early but partial recovery to nerve injury (Fig. 9). In contrast, mice exposed to local Rb siRNA had a more rapid recovery of hindpaw grip strength, earlier resolution of mechanical hypoalgesia and prevention of thermal hyperalgesia. Regenerating axons exposed to Rb siRNA also had more rapid recovery of sensory but not motor conduction velocity. Taken together, these results provide evidence that $\mathrm{Rb}$ knockdown is associated with functional indices of regeneration following nerve injury. They support our working hypothesis that in vitro and early in vivo outgrowth indices of axon growth predict functional benefits of regenerative strategies.

\section{Discusssion}

An overriding conclusion from our work is its support for the concept that manipulation of tumour suppressor pathways offers unexplored potential to manipulate growth patterns in adult 
a

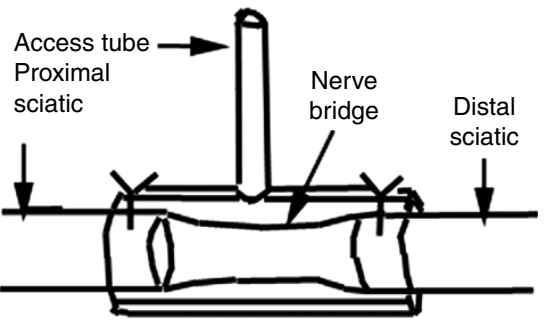

b

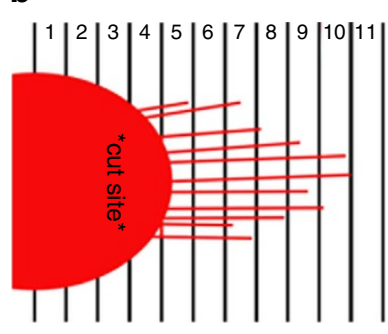

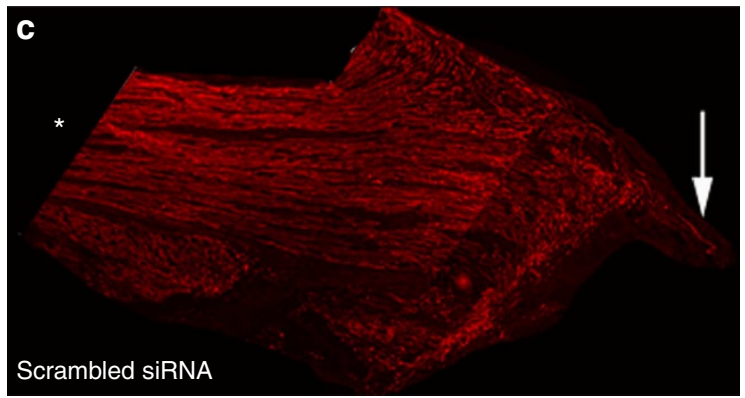
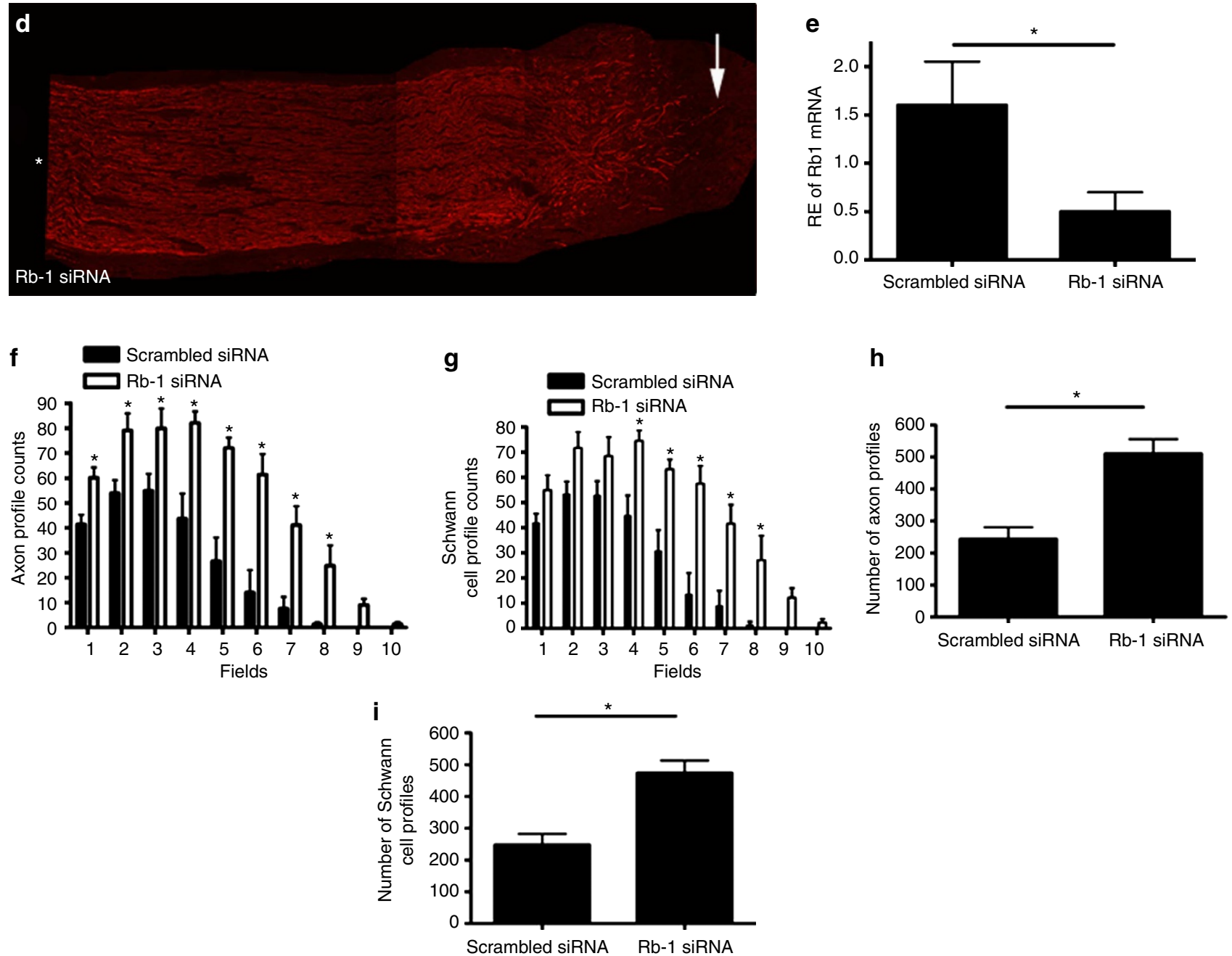

Figure 8 | Knockdown of Rb1 increases axon regeneration in vivo. Regeneration conduit showing where the proximal and distal sciatic nerves are sutured into the chamber (a). To the right is a schematic of the field quantification used for regenerating axon length (b). Longitudinal section of the sciatic nerve labelled with NF200, 7 days after treatment with scrambled siRNA in the regeneration chamber (c). Longitudinal section of sciatic nerve 7 days after treatment with Rb1 siRNA (asterisk denotes the side of the proximal stump, arrow points to the longest regenerating axon(d)) (for c, d Bar $=200 \mu \mathrm{m})$. qRT-PCR showed a decrease in Rb1 mRNA with Alexa-488 Rb1 siRNA in the chamber ( ${ }^{\star} P<0.05, n=5$; unpaired one-tailed Student's $t$-test) (e). Axon profile count in fields from the injury site $\left({ }^{\star} P<0.05, n=4\right.$; unpaired two-tailed Student's $t$-test) (f). Total axon counts for regenerating tip for scrambled and Rb1 siRNA ( ${ }^{\star} P<0.005, n=4$; unpaired two-tailed Student's $t$-test) $(\mathbf{h})$. Schwann cell profile counts from the injury site $\left({ }^{\star} P<0.05\right.$, $n=4$; unpaired two-tailed Student's $t$-test) $(\mathbf{g})$. Total Schwann cell counts for regenerating tip for scrambled and Rb1 siRNA $(P<0.005, n=4$; unpaired two-tailed Student's $t$-test) (i). Values are means \pm s.e.m.

neurons. In this context, $\mathrm{Rb}$ is a very well-known representative; however, its expression and role in adult neurons and their growth have not been previously explored. Our findings parallel how peripheral neurons respond to suppression of another tumour suppressor molecule, PTEN ${ }^{7}$. Both knockdown of $\mathrm{Rb}$ in this work and PTEN previously were associated with robust neurite outgrowth in vivo, particularly prominent in preconditioned neurons. 'Preconditioning' refers to the property of neurons to ramp up their regenerative activity in response to a prior lesion and is associated with substantial rises in outgrowth. Exploitation of preconditioned pathways has been a goal of regenerative investigators for some years, in part mimicked by 
a

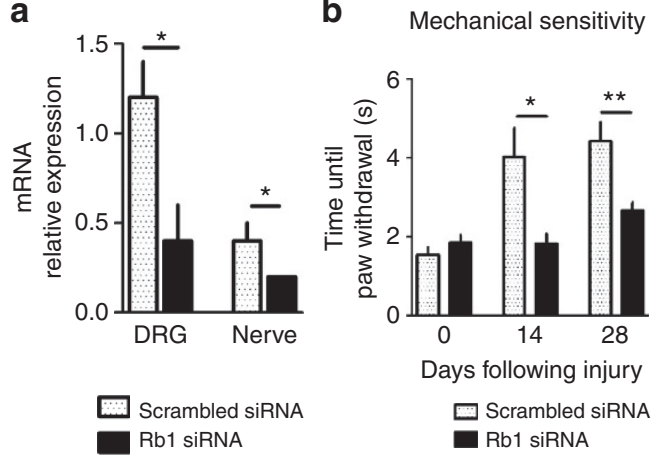

C

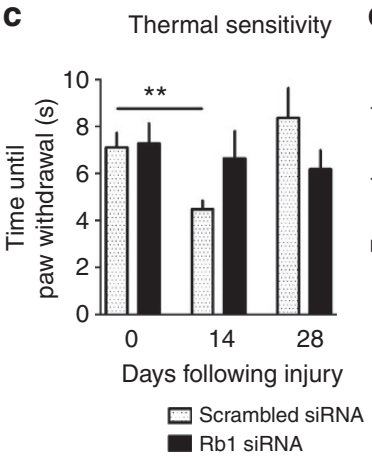

d Hindpaw grip strength $\mathbf{e}$

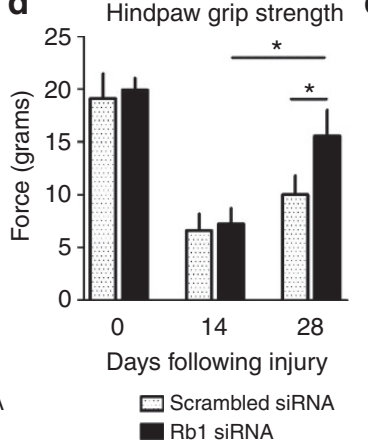

Conduction velocity of regenerating axons

Figure 9 | Local knockdown of Rb1 improves the functional recovery following nerve injury. Outbred mice underwent a crush injury of the sciatic nerve under anaesthesia. Immediately after the injury, and for 5 additional days, the injury site was administered either scrambled sequence siRNA or siRNA directed to Rb1. Rb1 knockdown was confirmed both at the level of the ipsilateral DRG housing the perikarya of the sciatic nerve sensory branches and locally at the injury site 7 days after injury (a) $\left({ }^{\star} P<0.05\right.$ for DRG; $n=7$ scrambled/DRG, four Rb siRNA/DRG; unpaired two-tailed Student's $t$-test; ${ }^{\star} P<0.05$ for nerve; $n=8$ scrambled/nerve, $4 /$ Rb1 siRNA/nerve; unpaired two-tailed Student's $t$-test with Welch's correction). Serial studies of mechanical sensitivity of the ipsilateral hindpaw indicated a rapid improvement in sensitivity in the Rb1 siRNA group at both 14 and 28 days following injury (b) $\left({ }^{\star} P<0.05\right.$ at 14 days and ${ }^{\star} P<0.005$ at 28 days; $n=8-10$; unpaired two-tailed Student's $t$-test). Thermal sensitivity studies identified injury-related hyperalgesia in the scrambled siRNA group at 14 days, but not the Rb1 siRNA group. By 28 days, both groups had recovered, but there was a nonsignificant trend towards more sensitivity in the Rb1 siRNA-treated group (c) ( ${ }^{\star} P<0.005 ; n=8-10$; unpaired two-tailed Student's $t$-test). After injury there was an expected decline in hindpaw grip power at 14 days. By 28 days, greater recovery in hindpaw power was identified in mice treated with Rb siRNA (d) ( ${ }^{\star} P<0.0514$ versus 28 days for Rb1 siRNA; $n=8=10$; unpaired two-tailed Student's $t$-test; ${ }^{*} P<0.05$ for 28 days; $n=8-10$; unpaired one-tailed Student's $t$-test). Multifibre sciatic-tibial motor conduction studies identified better recovery in sensory conduction velocity at 28 days post injury. No impact on motor conduction velocity was identified (e) $\left({ }^{\star} P<0.05\right.$ for sensory conduction velocity; $n=8-9$; unpaired one-tailed Student's t-test). Values are means \pm s.e.m.

rises in neuronal cAMP levels ${ }^{27}$. Remarkably, both PTEN and Rb suppression had impacts that were additive to preconditioning, an exciting form of plasticity that, if exploited, may have wide applicability. Both approaches also translated their impacts into benefits on outgrowth from transected peripheral nerve trunks.

We believe that the responses we identified to $\mathrm{Rb}$ knockdown or silencing were neuronal and were not mediated by an impact on glial cells. This was supported by the direct findings involving neurons in vitro, and Rb's very limited expression in glial cells. The retrograde knockdown of $\mathrm{Rb}$ we observed in DRGs after crush injury and local delivery of Rb siRNA suggests retrograde axonal transport of the siRNA in neurons.

Unlike PTEN knockdown, the mechanism of Rb knockdownmediated plasticity does not appear to involve upregulated pAkt. While this finding excludes a well-known neuron plasticity pathway, Sears and Nevins ${ }^{10}$ suggest that pAkt may instead operate upstream of $\mathrm{Rb}$. If that is the case, knockdown of $\mathrm{Rb}$ may have derepressed a constitutive action of pAkt on growth. Its robust actions however suggest a more active mechanism. Similarly, by acting upstream of Rb, MEK-ERK-Myc are less likely to be implicated in how Rb knockdown enhances plasticity. To search for other known targets of $\mathrm{Rb}$ that might instead mediate its actions, we identified upregulation of PPAR $\Upsilon$ in neurons. PPAR $\Upsilon$ antagonism blocked the plasticity associated with $\mathrm{Rb}$ knockdown and a PPAR $\Upsilon$ agonist increased neurite outgrowth. The involvement of PPAR $\Upsilon$, however, does not exclude the possibility that it acts parallel to other unexplored pathways involved in growth cone advancement. This is possible since the PPARY agonist alone had a less robust impact on both outgrowth but particularly on branching than $\mathrm{Rb}$ knockdown alone. Changes in PPAR $\Upsilon$ levels in response to $\mathrm{Rb}$ manipulation are described in other systems ${ }^{17,28,29}$. For example, PPAR $\Upsilon$ may render CNS neuroprotection ${ }^{30,31}$ and is expressed in injured sciatic nerves. The latter form of expression has been linked to growth on inhibitor myelin substrates ${ }^{20,32}$. Despite these findings, its role in adult nerve regeneration has not been otherwise examined. $\mathrm{Rb}$ inhibits $\mathrm{E} 2 \mathrm{~F}$ transcriptional activity where $\mathrm{E} 2 \mathrm{~F} 1$ and E2F3 appear to promote PPAR $\Upsilon$ transcription, whereas E2F4 represses $\mathrm{it}^{33,34}$. Thus under our experimental conditions, $\mathrm{Rb}$ silencing-mediated activation of E2F isoforms might have enhanced the expression of PPAR $Y$. Over threshold activation of E2F can direct cells to other fates also, leading to death of mature neurons by stimulating cell cycle entry ${ }^{22}$. Thus, the silencing of the $\mathrm{Rb}$ protein $(\sim 50 \%)$ achieved under our experimental conditions may represent optimal activation of E2F, prompting mature neurons to facilitate axon regeneration.

While the acceleration of outgrowth of both axons and SCs beyond their severed nerve trunks is a critical first step towards improving the outcome of these lesions, our data also suggest that this outgrowth enhances functional and longer-term regenerative outcomes. The potential role of $\mathrm{Rb}$ knockdown in central axon pathways has not yet been explored, sites with yet greater regenerative restraints. Despite this caveat, we believe that both $\mathrm{Rb}$ and PPAR $\Upsilon$ are important and novel targets for newer regenerative strategies. In addition to identifying two new molecular players in neuronal regeneration, our findings indicate the strong possibility that a wider range of novel shared oncogenic and neuronal proteins and pathways exist. Their manipulation in a localized and temporary context may offer opportunities to improve neurological outcome.

\section{Methods}

Animals and preconditioning lesion experiments. Adult male Sprague-Dawley rats (Charles River Laboratory, Senneville, Canada) with an initial weight of 200 $300 \mathrm{~g}$ were used and all animal studies were approved by the University of Calgary Animal Care Committee. For preconditioning, the sciatic nerve was cut at the mid-thigh level under aseptic conditions. A sham injury in separate animals was performed by exposing but not severing the sciatic nerve. Rb-floxed mice breeder pairs (FVB; $129-R b 1^{t m 2 B r n}$ ) were obtained from the National Cancer Institute mouse repository. The mice were propagated in the in-house animal care facility in the University of Calgary. For in vivo regeneration experiments and additional in vitro sensory neuron assays, we used male CD1 mice (Charles River Laboratory) with an initial weight of $17-21 \mathrm{~g}$. 
Quantitative reverse transcription-polymerase chain reaction. Total RNA was extracted from tissues using Trizol reagent and PCR primers that were designed using software Primer Express 2.0 (Applied Biosystems, Foster City, CA, USA):

Cyclophilin F $5^{\prime}$-TGTGCCAGGGTGGTGACTT-3'

R $5^{\prime}$-TCAAATTTCTCTCCGTAGATGGACTT- $3^{\prime}$

RPLP0 F $5^{\prime}$-TACCTGCTCAGAACACCGGTCT- $3^{\prime}$

R 5'-GCACATCGCTCAGGATTTCAA- ${ }^{\prime}$

Rb1 F 5'-TTACAGTATGCCTCCACCAGGC-3'

R $5^{\prime}$-TTACCTCCAGGAATCCGCAAG- ${ }^{\prime}$

PPARg (rat) F5'-TTCAAACTCCCTCATGGCCA-3'

R 5'-GTTCTTCGGAAAAAACCCTTGC-3'

PPARg (mouse) F5'-GATCATCTACACGATGCTGGCC-3'

R $5^{\prime}$-TTCCGCAGGCTTTTGAGGA- $3^{\prime}$

Cyclophilin, RPLP0 or Rb1 products were labelled with SybrGreen (Invitrogen, Burlington, Ontario, Canada). All reactions were performed in duplicate using an ABI PRISM 7000 Sequence Detection System (Applied Biosystems) and analysed using the $2^{\Delta \Delta}$ Cycle Threshold method. Results were presented as the fold induction of mRNA in L4-L5 DRG neurons or sciatic nerve normalized to Cyclophilin or RPLP0, and compared to normal, uninjured L4-L5 DRG neurons or sciatic nerve (defined as 1.0 -fold).

Western immunoblot. Ten micrograms of total protein, extracted from adult sensory neuronal cultures treated with scrambled siRNA or Rb siRNA, were loaded for SDS-PAGE. Membrane was incubated overnight with the respective antibodies: $\mathrm{Rb}, 1: 500$ in $2 \%$ bovine serum albumin (BSA) in TBST ( $2 \%$ TBST), rabbit polyclonal, Santa Cruz; PPAR, , 1:400 in 2\% TBST, mouse monoclonal, Santa Cruz; pAkt, $1: 500$ in 2\% TBST, rabbit monoclonal, Cell Signalling; pak1, $1: 1,000$ in $2 \%$ TBST, rabbit monoclonal, Abcam; cdk5; 1:500 in 2\% TBST; rabbit polyclonal; Santa Cruz. Tubulin (1:3,000 in 2\% TBST; mouse monoclonal, Sigma) and actin (1:1,000 in $2 \%$ TBST; mouse monoclonal, Millipore) were used as the loading controls. Quantification of bands was through Adobe Photoshop and the band densities were normalized with those of the loading control.

Antibodies and immunohistochemistry. Harvested L4, L5 DRGs and sciatic nerve (normal or proximal and distal to transection) were probed for $\mathrm{Rb}(1: 200$, Santa Cruz Biotechnology, sc-50), and double-labelled with S100 $\beta$ (1:200, Sigma), or with NF200 (1:400, Rabbit polyclonal, Sigma-Aldrich) for $48 \mathrm{~h}$, and then rinsed three times in phosphate-buffered saline. Secondary antibodies were sheep antimouse IgG CY3 conjugate (1:100, Sigma) and Alexa Fluor 488 goat anti-rabbit IgG $(\mathrm{H}+\mathrm{L})$ conjugate (1:500, Cedarlane, Horby, Canada). Dissociated DRG cultures were labelled with $\mathrm{Rb}$ (1:200), PPAR $\Upsilon$ (1:50, Mouse monoclonal, Cell Signalling), NF200 (1:500), activated caspase 3 (1:200); phospho-H2A.X (1:200); and $\beta$ tubulin III (1:100). Antibodies to activated caspase 3 and phosphohistone H2A.X (Ser 139) were purchased from Trevigen and Millipore, respectively. $\beta$ tubulin III antibody was purchased from Sigma.

Adult sensory neuron cultures. Dissociated adult sensory neuron culture protocols using rat DRG were as described previously ${ }^{7}$. Briefly, rats were anaesthetised with Isoflurane (Abbot Laboratories) and then killed 3 days following the conditioning lesion or sham surgery. L4-L5 DRGs were removed from the rats and placed into L15 (Invitrogen) medium (one rat (two DRGs) per condition per culture day were plated on a four-chamber slide). The DRGs were rinsed and then transferred to a tube containing $2 \mathrm{ml} 0.1 \%$ collagenase (Invitrogen)/L15. Following incubation at $37^{\circ} \mathrm{C}$, the DRGs were placed into single-cell suspension by triturating. The single-cell suspension was spun and washed three times in $2 \mathrm{ml}$ L15. After the final spin, the cells were resuspended in L15 and passed through a $70-\mu \mathrm{m}$ mesh (VWR International Co.) and then placed in $500 \mu \mathrm{l}$ L15 enriched with 1:100 dilution of N2 supplement (Invitrogen) and $0.1 \%$ BSA (Sigma) and placed into a culture medium of Dulbecco's Modified Eagle Medium/F12 (DMEM/F12; Invitrogen) $+1: 100$ dilution $\mathrm{N} 2,0.5-0.8 \% \mathrm{BSA}$ and $0.2 \mathrm{ng} \mathrm{ml}^{-1} \mathrm{NGF}$ (Cedarlane Laboratories) plus 50U Penicillin $\times \mathrm{ml}^{-1}, 50 \mathrm{U}$ Streptomycin $\times \mathrm{ml}^{-1}$ (Invitrogen) and plated on poly-L-lysine (Sigma-Aldrich) and $10 \mu \mathrm{g} \mathrm{ml}^{-1}$ mouse laminin (Invitrogen)-coated plates. In separate experiments, Alexa-488-tagged $\mathrm{Rb}$ or scrambled siRNA (Qiagen, Valencia, CA, USA) were added to the medium at $20 \mathrm{nM}$ along with the HiPerFect transfection reagent according to the HiPerFect handbook (Qiagen). Cells were grown for 18-20 h and analysed for neurite extension (mean per neuron), number of primary neurites (defined as processes extending from the soma), length of the longest neurite, number of branches (defined as a branch point in a primary neurite) and cell body area quantified by the MetaXpress software and an observer blinded to their condition (Molecular Devices, Sunnyvale, CA, USA). Between 40 and 60 neurons were analysed per condition and per culture day (four culture days, 240 neurons total for each condition). Three-four rats/condition were routinely used. For siRNA experiments, neurons were quantified if they contained the Alexa 488 fluorescent siRNA tag. For PPAR $\Upsilon$ antagonist studies, the PPAR $\Upsilon$-specific antagonist GW9662 (Sigma), at 0.1 and $1 \mu \mathrm{M}$, was added to the culture with the Rb siRNA; neurite outgrowth studies were performed at $36 \mathrm{~h}$. For PPAR $\Upsilon$ agonist studies, the specific PPAR $\Upsilon$ agonist, $15 \mathrm{PGJ} 2$, at $100 \mathrm{nM}$, was added to the culture while plating the cells; neurite outgrowth studies were performed at $36 \mathrm{~h}$. The following Rb1 siRNA target sequences were used (Qiagen) for the in vitro work:

$5^{\prime}$-TAGCATATCTCCGACTAAATA-3' (rat studies, sequence 1)

$5^{\prime}$-AAGGAGCACGAGTGTAATGTA- ${ }^{\prime}$ (rat studies, sequence 2)

$5^{\prime}$-ATCGAGGTTCTCTGTAAAGAA-3' (mouse studies).

For E2F1, the siRNA target sequence (Qiagen) used was:

$5^{\prime}$-ACAAATGGTCATAGTGATCAA- ${ }^{\prime}$.

Adenovirus particles expressing GFP or Cre recombinase or both were

purchased from Vector Biolabs. Cisplatin was purchased from Sigma. Adult mice (FVB; 129-Rb1 ${ }^{t m 2 B r n}$; 6-weeks old) were anaesthetized using isoflurane and were killed by cardiac puncture. DRGs at the lumbar and thoracic regions were harvested from both sides of the spinal column. The isolated DRGs were placed in $0.1 \%$ collagenase and incubated at $37^{\circ} \mathrm{C}$ for $45 \mathrm{~min}$. The individual cells were then dissociated by repeated pipetting of the DRGs in collagenase solution followed by centrifugation ( 800 r.p.m.; $6 \mathrm{~min}$ ) to pellet down the cells. The cell suspension was then laid over $15 \%$ BSA and centrifuged to remove the debris formed as a middle layer. The cell pellet was suspended in culture media containing recommended growth factors and $50 \mathrm{U} \mathrm{ml}^{-1}$ penicillin and streptomycin. The cells were infected with either Ad-GFP or Ad-CMV-Cre particles (500 p.f.u. per cell) at the time of seeding in four-well chamber slides or coverslips precoated with poly-l-lysine and laminin. The cells were harvested at $24 \mathrm{~h}$ post infection and total mRNA was isolated. For neurite outgrowth studies and other immunocytochemical analyses, the cells were fixed at $48 \mathrm{~h}$ post infection. In separate experiments, the cells were infected with Ad-GFP-2A-iCre particles that express both Cre recombinase and GFP. Cisplatin was dissolved in saline and the primary sensory neuronal cells were incubated with $20 \mu \mathrm{g} \mathrm{ml}^{-1}$ cisplatin for $24 \mathrm{~h}$.

LDH toxicity assay. LDH release from sensory neurons in vitro was utilized as a measure of cell death (Sigma) according to the manufacturer's instructions. Briefly, the cell-culture supernatant and the LDH assay mixture were mixed in 1:2 ratios and incubated for $30 \mathrm{~min}$ at room temperature. The reaction was terminated using $1 \mathrm{~N} \mathrm{HCl}$ and the absorbance was read at $490 \mathrm{~nm}$. Absorbance at $690 \mathrm{~nm}$ was also read and used for the background correction.

Regeneration experiments. Our experimental design for analysing early outgrowth of axons from transected rat sciatic nerve has been previously described ${ }^{7,26}$ A $3-\mathrm{cm}$ incision was made along the length of the lateral aspect of the left thigh, beginning at the sciatic notch and ending near the knee. A second incision was made on the dorsal aspect of the animal superior to the scapulae and parallel to the vertebral column to facilitate insertion of a microinjection port (MIP). Next, blunt forceps were used to tunnel through the subdermal fascia between posterior and anterior incisions. The jaws of the forceps were then used to pull the catheter of the MIP from its implantation site to the incision at the hindlimb. The catheter of the MIP was glued into the access tube of the T-chamber using cyanoacrylate cement Blunt dissection was performed to expose and mobilize the left sciatic nerve, which was transected at the mid-thigh level using an 11-0 scalpel blade. The proximal and distal stumps of the nerve were then secured into the ends of the nerve chamber using a single 9-0 nylon (Ethicon) suture through the epineurium of each stump, leaving a gap $(3 \mathrm{~mm})$ between stumps. Before closing the wound, a single 4-0 silk suture (Ethicon) was applied to secure the T-intersection of the tube to underlying muscle and a second suture was used to reattach the retracted gluteal muscle. Finally, a continuous suture (4-0 silk) was used to close both incisions. At end point, nerve chambers were harvested by transection of proximal and distal nerve segments several millimetres from the nerve chamber. Removal of the epineurial sutures freed the regenerate, and the nerve bridge (regenerate) was obtained by lightly pulling the nerve through the chamber. Injections of $0.2 \mathrm{ml}$ were given on days $0,1,3$ and 5 for $\mathrm{Rb}$ and scrambled siRNA ( $2 \mu \mathrm{g}$ per injection). siRNA target sequences were for rat (sequence 1) as listed above, under in vitro work. Bridges spanning sciatic transections were processed for immunohistochemistry and probed for NF200 (1:800) and GFAP (1:250). We counted profiles in a line perpendicular to the direction of the bridge in serial fields distally in high-power fields every $270 \mu \mathrm{m}$ until reaching the last field expressing the heavy-chain neurofilament protein, NF200 (neurofilament; $n=3$ per group). Counts were made with the observer blinded to the treatment group ${ }^{25}$

For longer-term regeneration experiments, mice were anaesthetised using inhaled 2\% isoflurane (Abbott Laboratories) administered through a ventilator in a breathing chamber, shaved and a $3-\mathrm{cm}$ incision was made just below the right hip along the sciatic notch. All three branches of the sciatic nerve were crushed using small blunt-tipped needle driver for $20 \mathrm{~s}$. A small square piece of parafilm was then placed under the crushed sciatic nerve to prevent the siRNA preparation from diffusing into surrounding tissues, and a small piece of surgical gel was placed directly under the injury site and directly on top of the injury site. Over a 20-min interval, $10 \mu \mathrm{l}$ of saline and siRNA (Rbl or scrambled) solution was applied every $5 \mathrm{~min}$ to the crush area through the surgical gel, totalling to $40 \mu$ l. siRNA target sequences were for mouse as listed above, under in vitro work.

siRNA scrambled or siRNA Rb1 (Qiagen) was mixed with HiPerFect

Transfection Reagent (Qiagen) for at least $20 \mathrm{~min}$ at room temperature. Saline was then added to the mixture and it was placed on ice until being administered. After each application of the solution, the nerve was covered with parafilm and the wound was covered with gauze. After 20 min of bathing the nerve in siRNA, the 
wound was closed with a 3-0 vicryl suture. Twenty microlitres of the siRNA solution was then injected transdermally into the hindpaw on the injured side. All animals were given analgesic Buprenorphine Jell-O $\left(0.5 \mathrm{mg} \mathrm{kg}{ }^{-1}\right)$ once daily for 3 days post injury, housed individually in the University of Calgary animal Care Facility with normal light and dark cycles and given free access to food and water. For 5 days post injury, the mice were injected with $40 \mu \mathrm{l}$ of the siRNA solution through the sutures into the nerve injury site, and $20 \mu \mathrm{l}$ transdermally into the hindpaw under isoflurane induced anaesthesia. For some of the mice $(n=6$ scrambled sequence, $n=7 \mathrm{Rb}$ siRNA), injured sciatic nerve and ipsilateral L4-L6 DRG were harvested at 7 days and placed in Trizol reagent for qRT-PCR analysis to verify Rbl knockdown. The remaining mice were used to assess behavioural and electrophysiological recovery of innervation in the hindpaw. As detailed in previous work, we measured the recovery of hindpaw sensitivity to a hindpaw mechanical sensitivity, to thermal sensitivity and hindpaw grip power ${ }^{35}$. Mechanical sensitivity was measured using an automated Von Frey apparatus (dynamic plantar anesthesiometer, UGO Basile). A filament with a progressively increasing force $\left(2 \mathrm{~g} \mathrm{~s}^{-1}\right)$ was applied to the plantar surface of the mice through the wired mesh until a withdrawal reflex. From three separate measurements in each mouse, the mean latency (time to withdraw) and amount of threshold force were calculated. For testing the recovery of thermal sensation, we used a radiant heat source applied individually to the middle of the hindpaw and the mean latency (s) to withdrawal was calculated from three separate measurements in each mouse. Recovery of multifibre motor and sensory conduction in the hindpaw was also assessed as previously described ${ }^{35}$. Recordings were carried out in sciatic-tibial fibres in mice anaesthetised with isofluorane, at a near-nerve subcutaneous temperature of $37^{\circ} \mathrm{C}$, maintained by a thermosensitive heat lamp with supramaximal stimuli were delivered to the sciatic nerve at the notch and knee. Conduction velocities were calculated in motor axons between the notch and knee and in sensory axons between the knee and paw.

Analysis. Statistical analysis using one-way ANOVA with Tukey post hoc analysis was performed for qRT-PCR and neurite extension data. When only two experimental groups were compared, a Student's $t$-test (paired when appropriate for concurrent data; Welch's correction for unequal variances and nonparametric tests as appropriate; two-tailed unless otherwise specified; one-tailed when direction of outcome predictable) was used.

\section{References}

1. McDonald, D. et al. Early events of peripheral nerve regeneration. Neuron. Glia. Biol. 2, 139-147 (2006).

2. Zochodne, D. W. Neurobiology of Peripheral Nerve Regeneration (Cambridge, UK, 2008).

3. Noble, J. et al. Analysis of upper and lower extremity peripheral nerve injuries in a population of patients with multiple injuries. J. Trauma 45, 116 (1998).

4. Brushart, T. M. Nerve Repair (Oxford, UK, 2011).

5. Witzel, C., Rohde, C. \& Brushart, T. M. Pathway sampling by regenerating peripheral axons. J. Comp. Neurol. 485, 183 (2005).

6. Cheng, C. et al. Activated RHOA and peripheral axon regeneration. Exp. Neurol. 212, 358 (2008).

7. Christie, K. J. et al. PTEN inhibition to facilitate intrinsic regenerative outgrowth of adult peripheral axons. J. Neurosci. 30, 9306 (2010).

8. Ma, C. H. E. et al. Accelerating axonal growth promotes motor recovery after peripheral nerve injury in mice. J. Clin. Invest. 121, 4332-4347 (2011).

9. Jones, D. M. et al. The synergistic effects of NGF and IGF-1 on neurite growth in adult sensory neurons: convergence on the PI 3-kinase signaling pathway. J. Neurochem. 86, 1116 (2003).

10. Sears, R. C. \& Nevins, J. R. Signaling networks that link cell proliferation and cell fate. J. Biol. Chem. 277, 11617 (2002).

11. Andrusiak, M. G. et al. The retinoblastoma protein is essential for survival of postmitotic neurons. J. Neurosci. 32, 14809 (2012).

12. McClellan, K. A. et al. Unique requirement for $\mathrm{Rb} / \mathrm{E} 2 \mathrm{~F} 3$ in neuronal migration: evidence for cell cycle-independent functions. Mol. Cell Biol. 27, 4825 (2007).

13. Jacks, T. et al. Effects of an Rb mutation in the mouse. Nature 359, 295-300 (1992).

14. Cheng, C. \& Zochodne, D. W. Sensory neurons with activated caspase-3 survive long-term experimental diabetes. Diabetes 52, 2363-2371 (2003).

15. Sosa-Garcia, B. et al. A role for the retinoblastoma protein as a regulator of mouse osteoblast cell adhesion: implications for osteogenesis and osteosarcoma formation. PLoS ONE 5, el3954 (2010).

16. Alexander, K., Yang, H. S. \& Hinds, P. W. Cellular senescence requires CDK5 repression of Rac1 activity. Mol. Cell Biol. 24, 2808 (2004).

17. Calo, E. et al. $\mathrm{Rb}$ regulates fate choice and lineage commitment in vivo. Nature 466, 1110-1114 (2010).

18. Torre-Ubieta, L. et al. A FOXO-Pak1 transcriptional pathway controls neuronal polarity. Genes Dev. 24, 799 (2010).

19. Kanungo, J. et al. Targeting Cdk5 activity in neuronal degeneration and regeneration. Cell Mol. Neurobiol. 29, 1073 (2009).
20. Dill, J. et al. A molecular mechanism for ibuprofen-mediated RhoA inhibition in neurons'. J. Neurosci. 30, 963 (2010).

21. Singh, B. et al. Resistance to trophic neurite outgrowth of sensory neurons exposed to insulin'. J. Neurochem. 121, 263-276 (2012).

22. Futatsugi, A. et al. Cyclin-dependent kinase 5 regulates E2F transcription factor through phosphorylation of Rb protein in neurons. Cell Cycle 11, 1603 (2012).

23. Toth, C. C. et al. Locally synthesized calcitonin gene-related Peptide has a critical role in peripheral nerve regeneration. J. Neuropathol. Exp. Neurol. 68, 326 (2009).

24. Webber, C. A. et al. Schwann cells direct peripheral nerve regeneration through the Netrin-1 receptors, DCC and Unc5H2. Glia 59, 1503 (2011).

25. Chen, Y. Y. et al. Axon and Schwann cell partnership during nerve regrowth J. Neuropathol. Exp. Neurol. 64, 613 (2005).

26. McDonald, D. S. \& Zochodne, D. W. An injectable nerve regeneration chamber for studies of unstable soluble growth factors. J. Neurosci. Methods 122, 171 (2003).

27. Cao, Z. et al. The cytokine interleukin- 6 is sufficient but not necessary to mimic the peripheral conditioning lesion effect on axonal growth. J. Neurosci. 26, 5565 (2006).

28. Fajas, L. et al. The retinoblastoma-histone deacetylase 3 complex inhibits PPARgamma and adipocyte differentiation. Dev. Cell 3, 903 (2002).

29. Mercader, J. et al. Haploinsufficiency of the retinoblastoma protein gene reduces diet-induced obesity, insulin resistance, and hepatosteatosis in mice. Am. J. Physiol. Endocrinol. Metab. 297, E184-E193 (2009).

30. Zhao, X. et al. Neuronal PPARgamma deficiency increases susceptibility to brain damage after cerebral ischemia. J. Neurosci. 29, 6186 (2009).

31. Zuhayra, M. et al. Activation of cerebral peroxisome proliferator-activated receptors gamma (PPARgamma) reduces neuronal damage in the substantia nigra after transient focal cerebral ischaemia in the rat. Neuropathol. Appl. Neurobiol. 37, 738 (2011).

32. Cao, Y. et al. Changes of peroxisome proliferator-activated receptor-gamma on crushed rat sciatic nerves and differentiated primary schwann cells. J. Mol. Neurosci. 47, 380-388 (2011)

33. Fajas, L. et al. E2Fs regulate adipocyte differentiation. Dev. Cell 3, 39 (2002).

34. Blais, A. \& Dynlacht, B. D. Hitting their targets: an emerging picture of E2F and cell cycle control. Curr. Opin. Genet. Dev. 14, 527 (2004).

35. Kan, M. et al. Glucagon-like peptide 1, insulin, sensory neurons, and diabetic neuropathy. J. Neuropathol. Exp. Neurol. 71, 494 (2012).

\section{Acknowledgements}

This work was supported by an operating grant from the Canadian Institutes of Health Research (FRN15686). A.K. is supported by a fellowship from the Hotchkiss Brain Institute, and Alberta Innovates, Health Solutions. B.S. was supported by a graduate studentship from Alberta Innovates, Health Solutions. D.Z. has been supported by the Alberta Heritage Foundation for Medical Research. Rb-floxed mice breeder pairs (FVB; 129-Rb1tm2Brn) were obtained from the National Cancer Institute (NCI) mouse repository.

\section{Author contributions}

K.J.C. helped to identify and formulate the research question, and performed, analysed and interpreted experiments, wrote a draft of the paper and edited the submitted versions. A.K. participated equally in the first authorship and performed, analysed and interpreted experimental data, suggested additional directions and contributed to the manuscript compilation and editing. J.A.M. performed, analysed and interpreted data and made intellectual contributions to the work. B.S. performed and analysed experimental data and made intellectual contributions to the work. K.P. performed and revised experimental approaches and data and made intellectual contributions to the work. S.E performed and revised experimental approaches and data and made intellectual con tributions to the work. D.Z. helped to identify and formulate the research question, guided and supervised the progress of the work, and revised, collated and wrote the final drafts of the submission.

\section{Additional information}

Supplementary Information accompanies this paper at http://www.nature.com/ naturecommunications

Competing financial interests: The authors declare no competing financial interests

Reprints and permission information is available online at http://npg.nature.com/ reprintsandpermissions/

How to cite this article: Christie, K. J. et al. Enhancing adult nerve regeneration through the knockdown of retinoblastoma protein. Nat. Commun. 5:3670 doi: $10.1038 /$ ncomms4670 (2014) 